\title{
Outage Probability Analysis for MRC in $\eta-\mu$ Fading Channels with Co-Channel Interference
}

\author{
David Morales-Jiménez, José F. Paris, and Angel Lozano
}

\begin{abstract}
Exact closed-form expressions are obtained for the outage probability of maximal ratio combining in $\eta-\mu$ fading channels with antenna correlation and co-channel interference. The scenario considered in this work assumes the joint presence of background white Gaussian noise and independent Rayleigh-faded interferers with arbitrary powers. Outage probability results are obtained through an appropriate generalization of the moment-generating function of the $\eta-\mu$ fading distribution, for which new closed-form expressions are provided.
\end{abstract}

Index Terms-Outage probability, $\eta-\mu$ fading, co-channel interference (CCI), maximal ratio combining (MRC).

\section{INTRODUCTION}

Diversity combining is a well-known strategy to mitigate the performance degradation caused by multipath fading and co-channel interference (CCI) in wireless systems [1]. For a communication link without CCI, maximal ratio combining (MRC) is the optimal combining technique in terms of maximizing the SNR at the output of the combiner. However, optimal combining in the presence of $\mathrm{CCI}$ is much more complex than MRC and typically requires covariance information about the CCI that may not be available. Therefore, in practice many wireless systems use MRC even in the presence of CCI.

The $\eta-\mu$ fading model considers a very general non-line-of-sight propagation scenario. By setting two shape parameters $\eta$ and $\mu$, this model subsumes the classical fading distributions as particular cases, e.g., Nakagami- $q$ (Hoyt), one-sided Gaussian, Rayleigh, and Nakagami- $m$. Because of its wider generality, the $\eta-\mu$ distribution can better fit experimental data. A detailed description of the $\eta-\mu$ fading model can be found in [2] and references therein.

Outage probability (OP) is a key performance metric for narrowband wireless systems [1, ch. 10]. Although considerable attention has been paid to its analysis, few results are found in the literature for $\eta-\mu$ fading. As an analytical hurdle, the general approach adopted in [1, ch. 10] is not applicable because the Gaussian characterization of the $\eta-\mu$ distribution is not circularly symmetric. An approximated result in integral form has been provided in [3] for suboptimal equal gain combining. Only recently have exact closed forms for the OP

D. Morales-Jiménez and A. Lozano are with the Department of Information and Communication Technologies, Universitat Pompeu Fabra, 08018 Barcelona, Spain (e-mails: d.morales@upf.edu, angel.lozano@upf.edu).

J. F. Paris is with the Department of Communications Engineering, University of Málaga, E-29071 Málaga, Spain (e-mail: paris@ic.uma.es).

The work of D. Morales and A. Lozano is supported by AGAUR (2009 SGR 70) and by the Spanish Ministry of Science and Innovation (Ref. CONSOLIDER-INGENIO CSD2008-00010 "COMONSENS"). The work of J. F. Paris was supported in part by the Spanish Government and FEDER project TEC2011-25473. in $\eta-\mu$ fading channels been unveiled, either with background noise only [4] or in interference-limited conditions (without background noise) [5]; no results are available with mixed background noise and interference. For the classical fading distributions some such results are available [6, 7]. In particular, [6] allows for both background noise and interference under Nakagami- $m$ fading for the desired signal and Rayleigh-faded interferers. In turn, [7] considers a single-antenna receiver under Hoyt fading.

The analysis in $[6,7]$ reveals that the OP involves a certain generalization of the moment generating function (MGF) of the fading distribution, referred to as the incomplete generalized MGF (IG-MGF). Given the broadness of the $\eta-\mu$ distribution and the usefulness of the IG-MGF, it is of great interest to obtain expressions for this generalization which is not currently available in the literature.

In this paper, new expressions are obtained for the IG-MGF of the $\eta-\mu$ distribution. By utilizing the IG-MGF results, closed-form expressions are derived for the OP of MRC with an arbitrary number of (possibly correlated) antennas in $\eta-\mu$ fading channels under any arbitrary mixture of background noise and CCI. This generalizes and unifies the findings in $[4,6,7]$.

\section{INCOMPLETE GENERALIZED MGF OF THE $\eta-\mu$ FADING DISTRIBUTION}

The IG-MGF has been found useful in the analysis of communication systems subject to interference [6,7]. This section introduces this generalization of the MGF and presents original expressions for the IG-MGF of the sum of $\eta-\mu$ variates, which are then applied to the OP analysis of MRC in $\eta-\mu$ fading channels.

\section{A. Definitions}

Definition 1 (Incomplete Generalized MGF): Consider a continuous random variable (RV) $X$ with $\operatorname{PDF} f_{X}(\cdot)$ and CDF $F_{X}(\cdot)$. The IG-MGF of $X$, if it exists, is defined as

$$
\mathcal{G}_{X}(a, b ; \zeta)=\int_{\zeta}^{\infty} x^{a} \mathrm{e}^{b x} f_{X}(x) d x
$$

where $^{2} b \in \mathbb{C}, a$ is a nonnegative integer, and $\zeta \in \mathbb{R}, \zeta \geq 0$.

Definition 1 includes, as particular cases, several important statistical functions associated with $X: \mathcal{G}_{X}(0,0 ; \zeta)$ is the complementary $\mathrm{CDF} ; \mathcal{G}_{X}(0, b ; 0)$ is the MGF; $\mathcal{G}_{X}(0, b ; \zeta)$ is the marginal MGF and $\mathcal{G}_{X}(a, b ; 0)$ is the generalized MGF.

\footnotetext{
${ }^{2}$ Although $b$ is only evaluated on the real line, the complex domain is assumed here in accordance with the usual definition of the MGF in the context of communications theory [1].
} 
Definition 2 (Complementary IG-MGF): The complementary IG-MGF $\tilde{\mathcal{G}}_{X}(a, b ; \zeta)$ of a RV $X$ is, if it exists,

$$
\tilde{\mathcal{G}}_{X}(a, b ; \zeta)=\int_{0}^{\zeta} x^{a} \mathrm{e}^{b x} f_{X}(x) d x
$$

Definition 2 includes the CDF of $X$ as a particular case, specifically, $F_{X}(x)$ is given by $\tilde{\mathcal{G}}_{X}(0,0 ; x)$.

\section{B. IG-MGF of the Sum of Squared i.i.d. $\eta-\mu$ Variates}

Let us now focus on the RV $X=\sum_{i=1}^{L} Y_{i}^{2}$ where $Y_{i}^{2}$, $i=1, \ldots, L$, are independent and identically distributed (i.i.d.) squared $\eta-\mu$ variates with mean $\Omega=\mathrm{E}\left[Y_{i}^{2}\right] . X$ is the sum of $L$ i.i.d. squared $\eta-\mu$ variates and its MGF is given by [2]

$$
\mathcal{M}_{X}(s)=\left[\frac{c_{1} c_{2}}{\left(-s-c_{1}\right)\left(-s-c_{2}\right)}\right]^{\mu L},
$$

where $c_{1}=-\frac{2 \mu(h+H)}{\Omega}$ and $c_{2}=-\frac{2 \mu(h-H)}{\Omega}$. The parameters $h$ and $H$, functions of $\eta$, are defined as [2]

$\left\{\begin{array}{cccc}h=\frac{2+\eta^{-1}+\eta}{4}, & H=\frac{\eta^{-1}-\eta}{4} ; & 0<\eta<\infty & \text { Format 1, } \\ h=\frac{1}{1-\eta^{2}}, & H=\frac{\eta}{1-\eta^{2}} ; & -1<\eta<1 & \text { Format 2. }\end{array}\right.$

As explained in [2], the two different formats for the $\eta-\mu$ distribution correspond to different physical significances and range of values for $\eta$. The motivation for utilizing $h$ and $H$, rather than $\eta$, is to have a unified representation that encompasses both formats.

In order to obtain the IG-MGF of $X$ in closed-form, the subsequent Lemmas provide expressions for the generalized MGF, $\mathcal{G}_{X}(a, b ; 0)=\mathcal{G}_{X}(a, b ; \zeta)+\tilde{\mathcal{G}}_{X}(a, b ; \zeta)$, and for the complementary IG-MGF, $\tilde{\mathcal{G}}_{X}(a, b ; \zeta)$; their difference gives the final IG-MGF.

Lemma 1: Consider a RV $X$ given by the sum of $L$ i.i.d. squared $\eta-\mu$ variates with mean $\Omega$. Sufficient conditions for the existence of the generalized MGF of $X, \mathcal{G}_{X}(a, b ; 0)$, are: $a \geq 0$ and $\Re\{b\}<0$. Then, for arbitrary $\mu$,

$$
\begin{aligned}
& \mathcal{G}_{X}(a, b ; 0)=(-1)^{a}\left(c_{1} c_{2}\right)^{\mu L} a ! \\
& \quad \sum_{k=0}^{a} \frac{(-\mu L-k+1)_{k}(-\mu L-a+k+1)_{a-k}}{k !(a-k) !\left(-b-c_{1}\right)^{\mu L+k}\left(-b-c_{2}\right)^{\mu L+a-k}}
\end{aligned}
$$

where $(\cdot)_{a}$ is the pochhammer symbol.

Proof: See Appendix A.

Lemma 2: Consider a RV $X$ given by the sum of $L$ i.i.d. squared $\eta-\mu$ variates with mean $\Omega$. Then, the complementary IG-MGF of $X$ is given by

$$
\begin{aligned}
& \tilde{\mathcal{G}}_{X}(a, b ; \zeta)=\frac{\zeta^{2 \mu L+a}(-1)^{a}\left(c_{1} c_{2}\right)^{\mu L}}{\Gamma(2 \mu L+a+1)} \\
& \sum_{k=0}^{a} \frac{a !}{k !(a-k) !}(-\mu L-k+1)_{k}(-\mu L-a+k+1)_{a-k} \\
& \Phi_{2}^{(2)}\left[\mu L+k, \mu L+a-k ; 2 \mu L+a+1 ;\left(c_{1}+b\right) \zeta,\left(c_{2}+b\right) \zeta\right],
\end{aligned}
$$

where $\Gamma(\cdot)$ is the gamma function and $\Phi_{2}^{(2)}$ is the confluent Lauricella function [8].

Proof: See Appendix B.
Corollary 1: Under the conditions of Lemma 1 and Lemma 2, the IG-MGF of the sum of $L$ i.i.d. squared $\eta-\mu \mathrm{RVs}$ is given by

$$
\mathcal{G}_{X}(a, b ; \zeta)=\mathcal{G}_{X}(a, b ; 0)-\tilde{\mathcal{G}}_{X}(a, b ; \zeta),
$$

where $\mathcal{G}_{X}(a, b ; 0)$ and $\tilde{\mathcal{G}}_{X}(a, b ; \zeta)$ are respectively given in (5) and (6).

\section{IG-MGF of the Sum of Squared Correlated $\eta-\mu$ Variates}

For the case of correlated $\eta-\mu$ variates, we restrict the analysis to $2 \mu$ integer. This entails little loss in generality as it includes the classical fading distributions (Hoyt, one-sided Gaussian, Rayleigh, and Nakagami-m) as particular cases.

Consider $X=\sum_{i=1}^{L} Y_{i}^{2}$ with $Y_{i}^{2}, i=1, \ldots, L$, identically distributed (not necessarily independent) squared $\eta-\mu$ variates with mean $\Omega=\mathrm{E}\left[Y_{i}^{2}\right]$. The $\mathrm{RV} X$ is the sum of $L$ identically distributed squared $\eta-\mu$ variates (for integer $2 \mu$ ) and its MGF has been recently given in [9] as

$$
\mathcal{M}_{X}(s)=\prod_{i=1}^{L}\left(1-2 \lambda_{i}^{A} s\right)^{-\mu}\left(1-2 \lambda_{i}^{B} s\right)^{-\mu},
$$

where $\lambda_{i}^{A}$ and $\lambda_{i}^{B}, i=1 \ldots L$, are, respectively, the eigenvalues of the matrices $\mathbf{A}$ and $\mathbf{B}$, defined as

$$
\mathbf{A}=\frac{-1}{2 c_{1}}\left(\begin{array}{cccc}
1 & \rho_{12} & \ldots & \rho_{1 L} \\
\rho_{12}^{*} & 1 & \ldots & \rho_{2 L} \\
\vdots & \vdots & \ddots & \vdots \\
\rho_{1 L}^{*} & \rho_{2 L}^{*} & \cdots & 1
\end{array}\right) ; \quad \mathbf{B}=\frac{c_{1}}{c_{2}} \mathbf{A}
$$

with $\left\{\rho_{i j}\right\}$ being the correlation coefficients between the complex Gaussians that underlie $Y_{i}$ and $Y_{j}$ [9].

Lemma 3: Consider $X$ given by the sum of $L$ identically distributed squared $\eta-\mu$ variates with mean $\Omega$. Then, for integer $2 \mu$, the generalized MGF of $\mathrm{X}$ is given by

$$
\begin{gathered}
\mathcal{G}_{X}(a, b ; 0)=(-1)^{a} \sum_{r_{1}+\ldots+r_{2 L}=a} \frac{a !}{r_{1} ! r_{2} ! \ldots r_{2 L} !} \\
\prod_{i=1}^{2 L}\left(2 \lambda_{i}\right)^{-\mu}\left(-\mu-r_{i}+1\right)_{r_{i}}\left(-b+\frac{1}{2 \lambda_{i}}\right)^{-\mu-r_{i}},
\end{gathered}
$$

where $\lambda_{i}=\lambda_{\frac{i+1}{2}}^{A}$ for $i$ odd and $\lambda_{i}=\lambda_{\frac{i}{2}}^{B}$ for $i$ even.

Proof: See Appendix A.

Lemma 4: Consider $X$ given by the sum of $L$ identically distributed squared $\eta-\mu$ variates with mean $\Omega$. Then, for integer $2 \mu$, the complementary IG-MGF of $X$ is given by

$$
\begin{gathered}
\tilde{\mathcal{G}}_{X}(a, b ; \zeta)=\frac{(-1)^{a} \zeta^{2 \mu L+a}}{\Gamma(2 \mu L+a+1)} \\
\sum_{r_{1}+\ldots+r_{2 L}=a} \frac{a !}{r_{1} ! r_{2} ! \ldots . r_{2 L} !}\left(\prod_{i=1}^{2 L}\left(-\mu-r_{i}+1\right)_{r_{i}}\left(2 \lambda_{i}\right)^{-\mu}\right) \\
\Phi_{2}^{(2 L)}\left[\mu+r_{1}, . ., \mu+r_{2 L} ; 2 \mu L+a+1 ;\left(b-\frac{1}{2 \lambda_{1}}\right) \zeta, . .,\left(b-\frac{1}{2 \lambda_{2 L}}\right) \zeta\right]
\end{gathered}
$$

where $\lambda_{i}$ is as in Lemma 3.

Proof: See Appendix B.

The closed-form expression for the IG-MGF in the correlated case is obtained from Corollary 1 by substituting (9) and (10) into (7). 


\section{Outage Probability Analysis}

The mathematical tools provided in the previous section are now used to obtain exact closed-form and easily computable expressions for the OP of MRC receivers in $\eta-\mu$ fading channels under Rayleigh CCI.

\section{A. Models}

Consider an $L$-antenna MRC receiver with perfect channel knowledge. The desired signal at every antenna is contaminated by $M$ CCI terms plus additive white Gaussian noise (AWGN) with zero mean and variance $\sigma^{2}$. The desired signal, with unit average power, and the interference signals, with arbitrary average powers $P_{i}, i=1, \ldots, M$, experience flat fading. The desired signal experiences $\eta-\mu$ fading, possibly non-independent across antennas, while the CCI terms are subject to independent Rayleigh fading.

Let $\mathbf{h}_{0}=\left[h_{01}, \ldots, h_{0 L}\right]^{T}$ and $\mathbf{h}_{i}=\left[h_{i 1}, \ldots, h_{i L}\right]^{T}$ denote, respectively, the channel gain vectors for the desired and the $i$ th interferer at the receiver. The entries $\left\{h_{i j}\right\}$ have normalized average power, i.e., $\mathrm{E}\left[\left|h_{i j}\right|^{2}\right]=1, i=0, \ldots, M, j=1, \ldots, L$. For convenience, we define $\mathrm{SNR}=1 / \sigma^{2}$ as the signal-tonoise ratio per antenna and $\mathrm{SIR}_{i}=1 / P_{i}$ as the per-antenna signal-to-interference ratio for the $i$ th interferer. Then, the received baseband signal vector $\mathbf{y}$ can be written as

$$
\mathbf{y}=\mathbf{h}_{0} b_{0}+\sum_{i=1}^{M} \frac{1}{\sqrt{\mathrm{SIR}_{i}}} \mathbf{h}_{i} b_{i}+\frac{1}{\sqrt{\mathrm{SNR}}} \mathbf{n}
$$

where $\mathbf{n}$ is the $L$-dimensional complex noise with zero mean and unit variance, and $b_{0}$ and $b_{i}$ are, respectively, the transmitted symbols from the desired and $i$ th interfering user, normalized such that $\mathrm{E}\left[\left|b_{0}\right|^{2}\right]=\mathrm{E}\left[\left|b_{i}\right|^{2}\right]=1$.

With MRC, the output signal is $x_{\mathrm{MRC}}=\mathbf{h}_{0}^{H} \mathbf{y}$ which, from (11), equals

$$
x_{\mathrm{MRC}}=\left\|\mathbf{h}_{0}\right\|^{2} b_{0}+\sum_{i=1}^{M} \frac{1}{\sqrt{\operatorname{SIR}_{i}}} \mathbf{h}_{0}^{H} \mathbf{h}_{i} b_{i}+\frac{1}{\sqrt{\mathrm{SNR}}} \mathbf{h}_{0}^{H} \mathbf{n} .
$$

The output instantaneous signal-to-interference-plus-noise ratio (SINR) is then $\gamma=\frac{X}{Z+1 / \mathrm{SNR}}$, where $X=\left\|\mathbf{h}_{0}\right\|^{2}=$ $\sum_{n=1}^{L}\left|h_{0 n}\right|^{2}$ is the effective power of the desired signal at the MRC output, and $Z$ the total instantaneous power of the interfering signals. It follows that $X$ is distributed as the sum of $L$ squared $\eta-\mu$ variables with unit mean and, therefore, $\mathrm{E}[X]=L$.

\section{B. Problem Formulation}

Let us divide the total number of interferers $M$ into $J$ groups with $n_{i}$ interferers in a given group, where every interferer in a group has the same average power $1 / \mathrm{SIR}_{i}$. It is shown in [6] that the corresponding OP is

$$
\begin{gathered}
P_{\text {out }} \doteq \operatorname{Pr}\left\{\gamma \leq \gamma_{0}\right\}=\underbrace{\int_{0}^{\frac{\gamma_{0}}{\mathrm{SNR}}} f_{X}(x) d x}_{P_{\text {out }}^{\star}}+\sum_{i=1}^{J} \sum_{j=1}^{n_{i}} \sum_{k=0}^{n_{i}-j} \sum_{\ell=0}^{k} \\
E_{i j} \frac{e^{\frac{\mathrm{SIR}_{i}}{\mathrm{SNR}} \mathrm{SIR}_{i}^{k}(-\mathrm{SNR})^{\ell-k}}}{\ell !(k-\ell) ! \gamma_{0}^{\ell}} \int_{\frac{\gamma_{0}}{\mathrm{SNR}}}^{\infty} x^{\ell} \mathrm{e}^{-\mathrm{SIR}_{i} \frac{x}{\gamma_{0}}} f_{X}(x) d x,
\end{gathered}
$$

where $\gamma_{0}$ is the outage threshold and $E_{i j}$ are given by [6]:

$$
E_{i j}=(-1)^{j-1} \sum_{\Omega_{A}} \prod_{k=1, k \neq i}^{J} \frac{\left(n_{k}+q_{k}-1\right) !}{q_{k} !\left(n_{k}-1\right) !} \frac{\operatorname{SIR}_{k}^{n_{k}} \operatorname{SIR}_{i}^{q_{k}}}{\left(\operatorname{SIR}_{k}-\operatorname{SIR}_{i}\right)^{n_{k}+q_{k}}}
$$

with $\Omega_{A}$ being the set of $J$-tuples satisfying $\Omega_{A}=$ $\left\{\left(q_{1}, \ldots, q_{J}\right): q_{k} \in \mathbb{N}, q_{i}=0, \sum_{k=1}^{J} q_{k}=j-1\right\}$, and $\mathbb{N}$ the set of non-negative integers.

The OP in (13) is expressed in terms of two incomplete integrals. To obtain a closed-form expression, we proceed to work these integrals out. The first one, $P_{\text {out }}^{\star}$, represents the OP in the interference-free case and is given by the CDF of $X$, which is directly $\tilde{\mathcal{G}}_{X}\left(0,0 ; \frac{\gamma_{0}}{\mathrm{SNR}}\right)$. The second one, representing the impact of the interference on the OP, consists of a linear combination of the incomplete generalized MGF of $X, \mathcal{G}_{X}(\cdot, \cdot ; \cdot)$. Specifically, the integrals therein can be readily identified with the IG-MGF as

$$
\int_{\frac{\gamma_{0}}{\mathrm{SNR}}}^{\infty} x^{\ell} \mathrm{e}^{-\mathrm{SIR}_{i} \frac{x}{\gamma_{0}}} f_{X}(x) d x=\mathcal{G}_{X}\left(\ell, \frac{-\mathrm{SIR}_{i}}{\gamma_{0}} ; \frac{\gamma_{0}}{\mathrm{SNR}}\right) .
$$

\section{Outage Probability Results}

1) i.i.d. fading: The final $\mathrm{OP}$ expression for $\mathrm{MRC}$ in $\eta-\mu$ fading channels under the presence of Rayleigh-faded interferers is obtained by substituting (15) into (13), which yields

$$
\begin{aligned}
& P_{\text {out }}=\tilde{\mathcal{G}}_{X}\left(0,0 ; \frac{\gamma_{0}}{\mathrm{SNR}}\right)+ \\
& \sum_{i=1}^{J} \sum_{j=1}^{n_{i}} \sum_{k=0}^{n_{i}-j} \sum_{l=0}^{k} E_{i j} \frac{e^{\frac{\mathrm{SIR}_{i}}{\mathrm{SNR}} \mathrm{SIR}_{i}^{k}(-\mathrm{SNR})^{\ell-k}}}{\ell !(k-\ell) ! \gamma_{0}^{\ell}} \mathcal{G}_{X}\left(\ell, \frac{-\mathrm{SIR}_{i}}{\gamma_{0}} ; \frac{\gamma_{0}}{\mathrm{SNR}}\right),
\end{aligned}
$$

where the coefficients $E_{i j}$ are given in (14), $\tilde{\mathcal{G}}_{X}\left(0,0 ; \frac{\gamma_{0}}{\operatorname{SNR}}\right)$ is the CDF of $X$, given by Lemma 2 , and $\mathcal{G}_{X}(\cdot, \cdot, \cdot)$ is the IG-MGF of the sum of $L$ i.i.d. squared $\eta-\mu$ variates directly obtained from Corollary 1 . Thus, the OP in (16) is, in essence, expressed in terms of the confluent Lauricella function. Simplified expressions for the CDF of $X$ can be found in [4] for integer or half-integer values of $L \mu$. Thus, by making use of $[4,(7)]$, the first term in (16) is expressed as

$$
\tilde{\mathcal{G}}_{X}\left(0,0 ; \frac{\gamma_{0}}{\mathrm{SNR}}\right)=1-Y_{L \mu}\left(\frac{H}{h}, \sqrt{\frac{2 h \mu \gamma_{0}}{\mathrm{SNR}}}\right),
$$

where $Y_{L \mu}$ represents Yacoub's integral [4]. Eqs. [4, (5)] and $[4,(6)]$, respectively, provide expressions for $Y_{L \mu}$ in terms of the Bessel and Marcum Q functions ( $L \mu$ half-integer) or the Jacobi polynomials ( $L \mu$ integer).

2) Correlated fading: The OP is given by (16), but in this case $X$ is the sum of $L$ correlated squared $\eta-\mu$ variates and, consequently, its $\mathrm{CDF}, \tilde{\mathcal{G}}_{X}\left(0,0 ; \frac{\gamma_{0}}{\mathrm{SNR}}\right)$, is obtained from (10), while its IG-MGF, $\mathcal{G}_{X}(\cdot, \cdot, \cdot)$, is obtained by using (9) and (10) in Corollary 1. Recall that (9) and (10) hold only for integer values of $2 \mu$ and, therefore, the analysis in the correlated case is restricted to such values (sufficient to model all the classical fading distributions). 


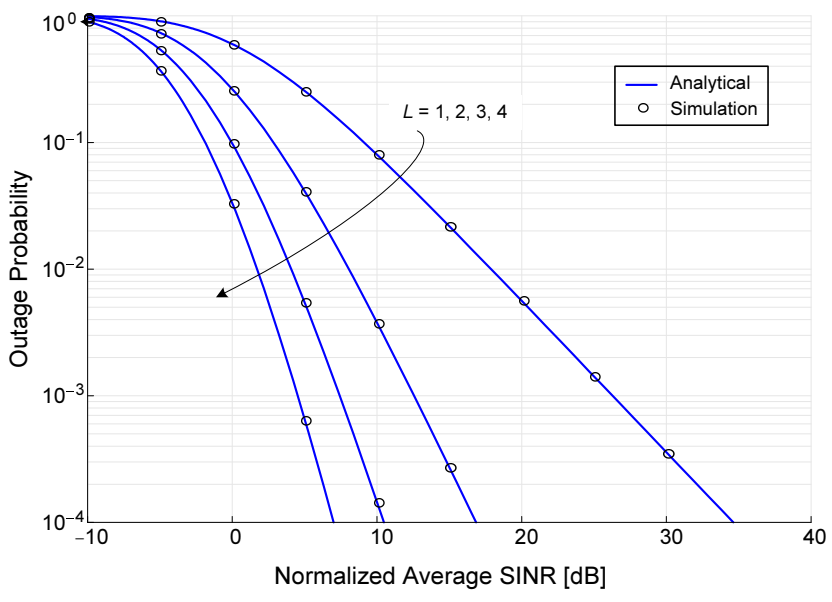

Fig. 1. OP versus average SINR normalized by $\gamma_{0}$ for MRC with $L$ independent antennas and fading parameters $\eta=0.3, \mu=0.6$.

\section{NumeriCAl Results}

This section presents numerical evaluations of the derived OP expressions validated against Monte-Carlo simulations entailing 10000 realizations per simulated point. The simulation model corresponds to the one described in Section III.

Figs. 1 and 2 show the OP of an $L$-antenna MRC receiver from (16), respectively for the i.i.d. and the correlated cases. The OP is plotted against the average SINR per antenna normalized by $\gamma_{0}$, i.e.,

$$
\frac{\bar{\gamma}}{\gamma_{0}}[\mathrm{~dB}]=10 \log _{10}\left(\frac{1}{\gamma_{0}\left(\sum 1 / \mathrm{SIR}_{i}+1 / \mathrm{SNR}\right)}\right),
$$

for several values of $L$ and different fading parameters $\eta$ and $\mu$. Three interferers are considered with $\mathrm{SIR}_{1}=6 \mathrm{~dB}$ and $\mathrm{SIR}_{2}=\mathrm{SIR}_{3}=9 \mathrm{~dB}$, while SNR $=10 \mathrm{~dB}$. Results in Fig. 1 correspond to i.i.d. fading and parameters $\eta=0.3, \mu=0.6$. Fig. 2 shows the OP for correlated fading with correlation coefficients $\rho_{12}=0.6, \rho_{13}=0.4$, and $\rho_{23}=0.2$. In this case, the fading parameters have been set to $\{\eta=2, \mu=0.5\}$, corresponding to Nakagami- $q$ (Hoyt) with $q=\sqrt{2}$, and to $\{\eta=1, \mu=1.5\}$, corresponding to Nakagami- $m$ with $m=3$. In both figures, the analytical results are in perfect agreement with the Monte-Carlo simulations.

\section{APPENDIX A}

\section{Proof of LEMMA 1 AND LEMMA 3}

The generalized MGF can be expressed as $\mathcal{G}_{X}(a, b ; 0)=$ $\left.\mathcal{L}\left[x^{a} f_{X}(x) ; s\right]\right|_{s=-b}$ with $\mathcal{L}$ the Laplace transform. Then, $\mathcal{M}_{X}(-s)=\mathcal{L}\left[f_{X}(x) ; s\right]$ and the differentiation property of the Laplace transform lead to

$$
\mathcal{G}_{X}(a, b ; 0)=\left.(-1)^{a} \frac{d^{a}}{d s^{a}}\left[\mathcal{M}_{X}(-s)\right]\right|_{s=-b} .
$$

Finally, (5) and (9) are obtained, respectively, by substituting (3) and (8) into (18), followed by application of the Leibniz rule and some algebra.

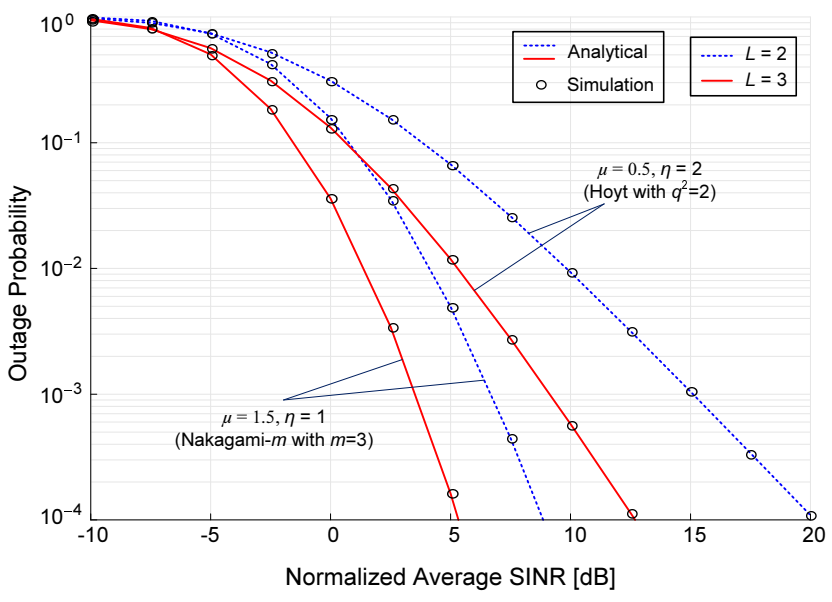

Fig. 2. OP versus average SINR normalized by $\gamma_{0}$ for MRC with $L$ correlated antennas.

\section{APPENDIX B \\ Proof of Lemma 2 AND Lemma 4}

The expressions for $\tilde{\mathcal{G}}_{X}(a, b ; \zeta)$ in (6) and (10), respectively for the i.i.d. and correlated cases, are obtained via the following rearrangement of the Laplace transform

$$
\mathcal{L}\left[\tilde{\mathcal{G}}_{X}(a, b ; t) ; t, s\right]=\frac{1}{s}(-1)^{a} \frac{d^{a}}{d s^{a}}\left[\mathcal{L}\left[f_{X}(t) ; s-b\right]\right] .
$$

Then, $\mathcal{L}\left[f_{X}(t) ; s-b\right]$ is replaced by $\mathcal{M}_{X}(-s+b)$, given in (3) and (8), and after some algebra, the resulting expression is identified with [10, (3.43.1.4)], yielding (6) and (10) for the i.i.d. and correlated cases.

\section{REFERENCES}

[1] M. K. Simon and M.-S. Alouini, Digital Communications over Fading Channels, 2nd ed. John Wiley, 2005.

[2] M. Yacoub, "The $\kappa-\mu$ distribution and the $\eta-\mu$ distribution," IEEE Antennas Propag., vol. 49, no. 1, pp. 68-81, Feb. 2007.

[3] A. Moraes, D. da Costa, and M. Yacoub, "An outage analysis of multibranch diversity receivers with cochannel interference in $\alpha-\mu, \kappa-\mu$, and $\eta-\mu$ fading scenarios," Wireless Personal Communications, pp. 1-17, 2012. [Online]. Available: http://dx.doi.org/10.1007/s11277-012-0513-x

[4] D. Morales-Jimenez and J. F. Paris, "Outage probability analysis for $\eta-\mu$ fading channels," IEEE Commun. Lett., vol. 14, no. 6, pp. 521-523, Jun. 2010.

[5] J. F. Paris, "Outage probability in $\eta-\mu / \eta-\mu$ interferencelimited scenarios," ArXiv e-prints, Mar. 2011.

[6] J. Romero-Jerez and A. Goldsmith, "Receive antenna array strategies in fading and interference: An outage probability comparison," IEEE Trans. Wireless Commun., vol. 7, no. 3, pp. 920-932, Mar. 2008.

[7] J. F. Paris and D. Morales-Jimenez, "Outage probability analysis for Nakagami- $q$ (Hoyt) fading channels under Rayleigh interference," IEEE Trans. Wireless Commun., vol. 9, no. 4, pp. 12721276, Apr. 2010.

[8] H. Exton, Multiple Hypergeometric Functions and Applications. New York: John Wiley \& Sons, 1976.

[9] V. Asghari, D. da Costa, and S. Aissa, "Symbol error probability of rectangular QAM in MRC systems with correlated $\eta-\mu$ fading channels," IEEE Trans. Veh. Technol., vol. 59, no. 3, pp. 1497-1503, Mar. 2010.

[10] A. P. Prudnikov and Y. A. Brychkov, Integrals and Series. Gordon and Breach Science Publishers, 1992, vol. 4. 